\title{
André Suarès lecteur de Stendhal. La querelle sur le style
}

\section{Paola Cattani}

\section{Q OpenEdition}

Journals

\section{Édition électronique}

URL : http://journals.openedition.org/rief/800

DOI : $10.4000 /$ rief. 800

ISSN : 2240-7456

\section{Éditeur}

Seminario di filologia francese

\section{Référence électronique}

Paola Cattani, «André Suarès lecteur de Stendhal. La querelle sur le style », Revue italienne d'études françaises [En ligne], 2 | 2012, mis en ligne le 15 décembre 2012, consulté le 19 avril 2019. URL : http:// journals.openedition.org/rief/800 ; DOI : 10.4000/rief.800

Ce document a été généré automatiquement le 19 avril 2019

\section{(c) (i) (9)}

Les contenus de la RIEF sont mis à disposition selon les termes de la Licence Creative Commons Attribution - Pas d'Utilisation Commerciale - Pas de Modification 4.0 International. 


\title{
André Suarès lecteur de Stendhal. La querelle sur le style
}

\author{
Paola Cattani
}

\section{NOTE DE L'ÉDITEUR}

Un premier état de cette recherche a été exposé à la Fondazione Primoli à Rome en mai 2010, dans le cadre du Séminaire international Lectures et lecteurs de Stendhal, organisé par Massimo Colesanti, Hélène de Jacquelot, Béatrice Didier et Marie-Rose Corredor.

1 André Suarès est, de tous les écrivains qui constituent le premier groupe de la Nouvelle Revue Française, un des plus méconnus et des moins étudiés: cet esseulé de la scène littéraire parisienne, comme il se plait à se définir, participe pourtant de manière significative au chantier de la NRF par la rubrique mensuelle qu'il y tient entre 1912 et 1914. Appelé à la NRF sur l'initiative de Gide et de Copeau, qui le soustraient à la Grande Revue de Rouché où il écrivait auparavant ${ }^{1}$, Suarès entame en avril 1912 sa Chronique de Caërdal, qu'il poursuit jusqu'à la brusque rupture d'avec le groupe en 1914 (la collaboration de Suarès avec la NRF ne sera à nouveau possible qu'à partir de 1926 et de manière irrégulière, sous la direction de Paulhan) ${ }^{2}$; les essais critiques qui composent cette Chronique relèvent tantôt de l'article journalistique, tantôt du texte littéraire poétique, mais ils se caractérisent tous par une vis polemica aiguë, qui n'hésite pas à toucher aux polémiques critiques en cours, voire même à entrer sur le terrain politique. C'est à un nœud crucial de cette Chronique que nous nous attacherons tout particulièrement ici, en examinant le long article consacré à Stendhal et livré en deux fois, dans les numéros de mai et de juin $1914^{3}$.

2 Bien que Suarès ait été appelé à la NRF en tant que spécialiste de $\mathrm{Pascal}^{4}$, son œuvre littéraire et critique est davantage et plus intimement marquée par Stendhal, auquel il ne cesse, tout au long de son itinéraire intellectuel, de se référer comme à un modèle et à un interlocuteur ${ }^{5}$. Les références à Stendhal parsèment les essais critiques de Suarès : pour 
les cerner dans leur intégralité, il faut prendre aussi en compte, à côté des écrits sur Stendhal, des articles qui concernent entre autres Racine, Montaigne ou Chateaubriand. Pour comprendre l'importance du modèle de Stendhal pour Suarès, il convient ensuite de se tourner également vers le Voyage du condottière, l'ensemble des textes concernant les nombreux voyages que Suarès effectua en Italie, en une quête stendhalienne de beauté Que représente Stendhal aux yeux de Suarès, et quelle lecture ce dernier avance-t-il de l'œuvre stendhalienne? Nous nous proposons d'essayer de répondre à ces questions à partir d'un point d'observation spécifique, à savoir celui qui concerne le débat sur le style de Stendhal. C'est en effet en défendant le style du maitre contre les critiques de ses détracteurs que Suarès fait ses débuts de stendhalien.

Dans un article de 1919 (et inséré par la suite dans Xénies, recueil de 1923), Suarès s'enorgueillit d'avoir été le premier à défendre un style souvent contesté : « Le premier, je pense, il y a déjà dix et onze ans, j'ai défendu Stendhal de mal écrire. Bien plus, en dépit des docteurs et des académiciens, j'admirais en lui un prince du langage $»^{7}$. Suarès fait ici allusion à un chapitre du Voyage du condottière intitulé "Stendhal en Lombardie », où il s'était effectivement attaché à célébrer les qualités d'un style décrit comme étant

d'acier, de la pointe la plus acérée et la plus fine. Ni images, ni périodes. Ni la lyre, ni l'éloquence. Il est nu comme la ligne. Il me rappelle Lysias et l'orateur attique, si les Athéniens, au lieu de plaider, faisaient l'analyse de l'homme. Pour tout dire, il est Grec. Chaque phrase de Stendhal est pleine de sens, et d'un feu clair, qui fait de la lumière, sans chaleur. Toutes ces phrases ensemble tombent comme des étincelles : ceux qui ne sont pas sensibles à ce feu d'intelligence, diront qu'elles tombent comme la pluie. ${ }^{8}$

Si ce morceau a bien été composé avant 1910 , comme Suarès le prétend ${ }^{9}$, il est loin d'être le premier ni surtout le seul à se dresser contre les critiques envers le style de Stendhal. $\mathrm{Au}$ cours des mêmes années, nombreux sont en effet les plaidoyers prononcés en sa défense. Gide, par exemple, dans son Journal, revient à plusieurs reprises à partir de 1907 sur un style qu'il prend comme modèle et comme stimulant pour apprendre à écrire sans médiation, de façon spontanée : «Stendhal n'a jamais été pour moi une nourriture ; mais j'y reviens toujours. C'est mon os de seiche ; j'y aiguise mon bec $»^{10}$; «Besoin de relire Stendhal. Oser écrire sans ordre $»^{11}$; jugements que l'on retrouve dans ses écrits critiques, tels que le «Journal sans dates » tenu à la NRF, qu'il place sous le signe de Stendhal en disculpant de manière explicite ce dernier de toute accusation sur son manque de lyrisme ${ }^{12}$. La préface de Lamiel (1947) confirme finalement cette appréciation en réaffirmant qu'heureusement Stendhal, à l'encontre des conseils de Balzac, n'a pas corrigé son style, puisque «ce faisant, il allait à l'encontre de sa qualité majeure : le naturel $»^{13}$. Rivière, de son côté, remercie Gide de l'avoir obligé à lire Stendhal : «Je sens son style me faire du bien $»^{14}$; Remy de Gourmont, contribuant à la même époque à consolider le mythe de Stendhal, publie en 1912 une défense de son «style et art " $^{15}$. L'appréciation positive de l'écriture inspirée du «Code civil » sera ensuite reprise et approfondie notamment par Alain, qui s'exprime là-dessus au début des années 1920 dans ses Propos ainsi que dans ses différents articles sur Stendhal ${ }^{16}$, et par Jean Prévost qui s'attachera en particulier à montrer la complexité d'un style estimé, erronément à son avis, comme facile ${ }^{17}$.

5 Si l'observation de Suarès n'est donc pas isolée, il importe d'en préciser la signification et les enjeux. D'abord, comment est-il en effet possible qu'un fils du symbolisme, un poète qui a accompli sa formation sous le signe de la poésie symboliste et qui consacre la plus grande partie de sa production à la poésie, comble de louanges une prose qui se vante de n'être pas soignée du point de vue de la forme? Et ensuite, si les attaques livrées par 
Stendhal contre le style rhétorique et ampoulé se situent, comme la critique a pu le montrer ${ }^{18}$, dans la bataille à la fois politique et esthétique qu'il a menée contre un certain romantisme, et si elles apparaissent imbibées de l'idéologie post-révolutionnaire, comment ses propos, avec la polémique qui s'y rattache, sont-ils revivifiés et peuvent-ils trouver une actualité nouvelle au début du XXe siècle?

De Victor Hugo, qui méprise Stendhal lequel, selon lui, écrit « en patois » ${ }^{19}$, en passant par Flaubert, qui affirme n'avoir «jamais rien compris à l'enthousiasme de Balzac pour un semblable écrivain, après avoir lu le Rouge et le noir ${ }^{20}$, et jusqu'à Pierre Louÿs, qui, selon le témoignage de Valéry, insulte "cette prose intolérable ${ }^{21}$, le discrédit jeté sur le style de Stendhal fait l'objet d'une longue tradition critique, même parmi les écrivains. Au tout début du siècle, la prose de Stendhal devient la cible de l'analyse et de l'attaque d'Antoine Albalat, qui, dans son Travail du style enseigné par les corrections manuscrites des grands écrivains ${ }^{22}$, s'attache à corriger même des extraits du Rouge et le noir, dans ce qu'il considère comme les fautes stylistiques de Stendhal, à savoir les répétitions, les banalités etc. Cette approche revient sur des griefs exprimés également par une certaine critique stendhalienne, telle que celle de Cordier ou de Chuquet qui, dans les dernières années du siècle, s'accordent sur la « sécheresse » de Stendhal et sur son incapacité à « travailler la phrase $»^{23}$. Le discours de Suarès ne s'oppose pas seulement à ces critiques générales, il répond également à des accusations circonscrites. Sa défense s'organise principalement autour de trois points, qui ont tous trait au rapport entre le style et la rhétorique, et qui vont à l'encontre de la ligne interprétative qui, en dénonçant les insuffisances de style de Stendhal, met tout particulièrement en cause son refus de la rhétorique et du style élevé.

7 Dans le Voyage du condottière de 1910, Suarès défend d'abord l'écriture de Stendhal, d'un côté en opposant sa netteté à l'emphase, de l'autre en mettant sa clarté sur le compte du classicisme et de ses vertus, et non pas d'une nouveauté stylistique : "Cet homme de la Révolution, le plus opposé par l'esprit qui se puisse à Bossuet, écrit la langue la plus nette et la moins nouvelle. Plaidant contre Racine pour Shakespeare, il est plus classique que personne. [...] Il n'est pas un écrivain qui ne soit emphatique près de Stendhal $»^{24}$. Dans le même sens, Suarès aura bientôt soin de distinguer entre le refus de la rhétorique et l'attention portée à la forme. Dans l'article de 1919, en constatant le manque de sublime chez Stendhal (« rien ne [lui] manque, si ce n'est la couleur de la grande poésie »), Suarès observe en même temps : « D'ailleurs, Stendhal connaît le tourment de la forme [...]. Il sait la valeur d'un mot mis en sa place. Il a le sens de l'épithète $\aleph^{25}$, ce qui fait penser au passage de la lettre à Balzac où Stendhal précise : «Souvent je réfléchis un quart d'heure pour placer un adjectif avant ou après un substantif $»^{26}$. Enfin, troisième argument utilisé pour la défense de Stendhal, Suarès établit une corrélation entre un style net et la sincérité : «Stendhal n'a pas le plus beau style, mais le plus vrai ${ }^{27}$. La clarté est une vertu de l'âme qui coïncide avec la spontanéité; observation qui se renouvelle notamment sous la plume de Gide, lequel, comme on l'a vu, se tourne vers Stendhal précisément comme vers un modèle d'écriture de vie.

8 Cette défense revient sur des arguments que l'on peut retrouver en particulier dans les articles stendhaliens parus à la charnière des deux siècles dans la Revue Blanche et dans l' Ermitage. La lecture que Suarès donne de Stendhal doit beaucoup aux jugements exprimés sur l'auteur de la Chartreuse dans les pages de ces revues, par rapport auxquelles il convient de la situer.

9 Tout d'abord, c'est la Revue Blanche qui offre en 1901 une contribution remarquable au débat sur le style en publiant des extraits des marginalia de Stendhal pour la correction de 
la Chartreuse, suivant en cela le conseil de Balzac ${ }^{28}$. Il s'agit d'un ensemble de notations assez brèves, choisies et réunies par Pierre Brun; elles insistent sur l'aversion envers le style "noble et enflé », récusé en tant qu'associé à la "pauvreté du cœur», et sur la recherche de la clarté et de l'intelligibilité. « Par amour de la clarté et de l'intelligible j'ai été conduit au style qui est le contraire du style un peu enflé des romans actuels »: Stendhal estime que le style «noble » ne sera plus guère lisible au bout de quelque temps, et se donne pour mission d'" augmenter la clarté ", en s'opposant au style "élégant ", aussi bien qu'à celui "emphatique et philosophique» qui, plus qu'expliquer et transmettre, demandent à son avis de «croire». La Revue Blanche, qui par ailleurs offre une contribution fondamentale au stendhalisme dans la mesure où elle reproduit assez régulièrement des inédits ${ }^{29}$ et accueille dans ses pages les premiers articles de Léon Blum sur Stendhal ${ }^{30}$, réunit ici une sorte de bréviaire, de petit traité sur le style stendhalien, en se faisant surtout l'écho de la bataille livrée par Stendhal contre la rhétorique et les modèles littéraires qui en découlent.

Quelques années plus tard, c'est Paul Léautaud à l'Ermitage qui offre une contribution essentielle à cet égard. Dans son célèbre article de 1905 sur le Stendhal-Club, il insiste précisément sur les mérites de ce style non littéraire mais plutôt « d'affaires ", comme il le dit: « Rien n'a plus de prix pour moi que la netteté, la concision, et c'est si difficile de n'être pas littéraire, le premier mérite, à mes yeux. On a même beau faire, on l'est toujours un peu, de même qu'on écrit toujours plus de mots qu'il n'en faut. Savoir bien écrire mal, dis-je quelquefois $»^{31}$, jugement qu'il avait déjà exprimé quelques années auparavant dans son Journal ${ }^{32}$. Léautaud trace un chemin que Suarès empruntera lui aussi par la suite, en célébrant les qualités non littéraires de l'écriture stendhalienne : «Adieu ce qu'on appelle les règles littéraires, comme s'il y avait des règles pour écrire. Adieu les ronrons, les belles périodes, les tableaux calculés, les métaphores dont certains s'enchantent, les mots choisis jusqu'à l'étrangeté, tout le toc et tous les trucs littéraires. "L'horreur du vague autant que du niais et du pompeux", qu'avait à un si haut degré Stendhal, y éclate à chaque page $»^{33}$. Il propose d'ailleurs une comparaison qui reviendra sous la plume de Suarès, celle qui oppose Stendhal à La Bruyère : quoique ce dernier puisse être considéré comme un modèle de style clair et vif, il s'écoute cependant trop parler, et s'admire excessivement ${ }^{34}$.

11 La "Chronique Stendhalienne» que Léautaud tient avec Remy de Gourmont sous le pseudonyme de Coffe dans la même revue, à partir du mois de mai 1905, offre également des matériaux sur lesquels Suarès méditera sans aucun doute: c'est probablement à partir de ces pages qu'il pourra par exemple développer dans son Voyage du condottière une comparaison qui n'est ici qu'esquissée, celle entre Goethe et Stendhal en Italie, l'un à la recherche de ses illusions, l'autre sur la trace de la vérité de ses émotions ${ }^{35}$. Cette rubrique, qui se propose de faire régulièrement le point sur les publications et sur le débat concernant Stendhal, insiste davantage sur la question du style, en reportant souvent les jugements critiques émis à cet égard, favorables ou pas. Elle consacre par exemple certaines de ses notices à l'appréciation négative de Victor Hugo sur le " patois » de Stendhal et aux oppositions que Stendhal lui-même établit, à propos du style, entre Bossuet ou Chateaubriand et lui; quant au débat d'actualité, elle signale sur un ton polémique la tentative d'Albalat de réécrire la Chartreuse, ainsi que les critiques formulées par Faguet et d'autres ${ }^{36}$.

12 En développant donc sa défense du style dans le sillage du débat de ces revues, Suarès revient d'abord sur certaines oppositions établies par Stendhal et récemment soulignées 
par la critique, celle entre Stendhal et Bossuet par exemple (dont l'éloquence est réduite à une "blague sérieuse $»^{37}$ ), et bien entendu, celle entre Stendhal et Chateaubriand (il faut percer « les bulles de cette rhétorique sentimentale et de cette folle vanité $\left.{ }^{38}\right)$. Loin de revivifier ces seuls jugements critiques de Stendhal contre la rhétorique, Suarès lui emprunte d'ailleurs toute une géographie critique en matière d'histoire littéraire, en se faisant l'écho par exemple des appréciations exprimées sur l'Arioste et sur le Don Quichotte, ainsi que des moqueries à l'encontre de George Sand et de Musset, qui incarneraient le mauvais romantisme ${ }^{39}$, ou encore des critiques envers la perfection formelle de Racine (Suarès organise notamment son article de la NRF suivant les citations tirées de la Vie de Henry Brulard, et dont il donne en note les références précises ${ }^{40}$ ).

13 Mais à ces oppositions empruntées à Stendhal, Suarès ajoute, pour conduire son discours sur le style, deux parallélismes inédits: il associe, contre toute évidence, Stendhal à Verlaine et à Baudelaire. D'abord, parce que ces auteurs sont tous les trois des incompris, et qu'ils sont aimés par des happy few :

On sourit du mépris rieur que l'on voit à Stendhal pour Chateaubriand, se moquant du style moderne. Dans son antipathie, Stendhal quelque part semble avoir prévu Verlaine. Il n'est rien de si contraire à Verlaine que Stendhal. Lequel est le plus d'ici ? Et Stendhal croit aimer la musique! Mais quoi, la France qui a tant méconnu Stendhal, ne sait pas encore le prix de Verlaine. Or, depuis Dante, c'est le plus poète des poètes, le plus vrai, le plus pur, étant toujours en Dieu, et le plus musicien. ${ }^{41}$

Dans l'article «Stendhal, Verlaine, Gérard de Nerval et autres gueux ", Suarès inclut ces derniers dans la liste des écrivains maltraités par les critiques moralistes et vulgaires ${ }^{42}$. Ensuite, ils ont en commun un esprit libre et supérieur: comme Baudelaire, Stendhal « juge avec supériorité tous les auteurs, toutes les renommées, toutes les couronnes, fûtce Chateaubriand, fût-ce Racine $»^{43}$. Enfin, tous les trois récusent la fiction en faveur de la vérité et de la sincérité. Dans l'article qu'il consacre en 1917 à Verlaine, Suarès les associe en ce sens qu'il les considère capables de se livrer au "goût cruel de se connaître, cette soif de se déchirer, cette curiosité des profondeurs » qui caractérisent la poésie véritable ${ }^{44}$ . Dans les mêmes pages, Suarès peut ainsi attribuer au style de Verlaine une qualité qui semble découler de cette association à Stendhal : il serait « le moins rhéteur des poètes, étant le plus musicien »; inversement, Stendhal, avec son style " décharné », devient « le moins lyrique des hommes, et pourtant des plus poètes $»^{45}$.

En réalité Suarès n'est pas le seul à établir ce lien entre l'auteur du Rouge et le noir et les poètes phares de sa génération. Gide également, qui par ailleurs insiste lui aussi sur l'opposition entre le mensonge « sincère " de Stendhal et celui fautif de Chateaubriand ${ }^{46}$, établit plus d'une fois un parallèle entre Stendhal et Baudelaire : il les associe notamment dans la préface à Armance de 1921 ainsi que dans l'article sur Rimbaud de 1941, eu égard au modèle de littérature "ésotérique " qu'ils incarnent; il évoque l'injuste déni qu'ils subissent de la part de leurs contemporains ${ }^{47}$, et la notion de "happy few " qu'ils contribuent à constituer ${ }^{48}$. Mais surtout Gide associe Stendhal et Baudelaire dans l'article de 1910 où il critique l'histoire littéraire de Brunetière et de Faguet : cet essai est d'autant plus significatif qu'il se trouve être à l'origine de la querelle qui éclate entre Gide et Suarès, en brouillant les rapports de ces deux écrivains liés depuis 1908 par une estime réciproque qui, cependant ne se transformera jamais en une amitié véritable. En dénonçant dans ces pages la façon dont la critique de Faguet tout comme celle de Brunetière méconnaissent certains auteurs du XIX siècle, Gide mentionne non seulement le cas célèbre de Baudelaire, mais aussi celui de Stendhal : ces deux auteurs représentent au contraire à ses yeux «la plus admirable intelligence critique de [leur] époque », et 
incarnent la « perfection secrète » à laquelle est insensible Faguet, qui cherche «l'autre perfection seule, rhétorique, logique, oratoire $»^{49}$. Développant son attaque contre Brunetière, Gide écrit : «Je ressens pour Brunetière parfois presque autant d'admiration que Suarès même », phrase qui lui attire de vives protestations de la part de Suarès, lequel s'indigne surtout d'avoir été mentionné à propos de Brunetière et non pas, ce qui aurait été plus pertinent et moins incorrect à son avis, pour ses articles sur Stendhal ${ }^{50}$.

L'auteur de la Chartreuse trouve donc une place cruciale au sein de cet épisode de dialogue manqué entre les deux écrivains au sujet de positions en réalité convergentes, épisode qui non seulement est très significatif du rapport qui est le leur pendant plusieurs années, mais qui est également révélateur du débat littéraire en cours, et dont le pivot est l'opposition à la critique de Brunetière et de Faguet. C'est sans doute à la suite de cette controverse de 1910 avec Gide que Suarès entreprend de réunir ses vues sur Stendhal dans un article, qui sera publié dans la NRF en 1914 comme on l'a vu, mais dont l'écriture remonte au mois d'avril 1911, ce que Suarès a soin de préciser à la fin de l'essai. En effet, dans ces pages, Suarès règle ses comptes, en particulier avec Brunetière, son ancien maître : en s'attachant à laver Stendhal de l'accusation de «fatuité » et de «dandysme » ( ( Dans la réserve d'un homme, ses ennemis voient de la fatuité »" ), c'est le jugement de ce critique qu'il a à l'esprit, le Brunetière qui en 1887 pouvait écrire, en corrigeant une dissertation de Romain Rolland, son élève à l'École Normale, camarade et ami intime de Suarès (ils étaient de la même promotion) : «Vous avez bien mis en relief un ou deux traits de la physionomie de Stendhal, mais vous auriez pu insister davantage sur la fatuité littéraire et son dandysme intellectuel. C'est que Stendhal est surtout un prétentieux personnage, et de tous les épicuriens le plus content de soi $»^{52}$. C'est à l'encontre des enseignements et de l'approche littéraire de celui qui a été longtemps son professeur que Suarès défend Stendhal et son style ${ }^{53}$; l'association avec Verlaine est révélatrice de sa bataille pour la défense de la poésie post-symboliste que Brunetière blâmait. Tout comme les remarques sur la "sincérité ", cette association témoigne également de l'adhésion de Suarès, au sein du post-symbolisme, à un certain vitalisme qui, tout en demeurant étranger au naturalisme, s'oppose au formalisme mallarméen.

Suarès se rallie ainsi à la lutte qui était déjà celle des deux revues qui avaient le plus contribué à l'engouement pour Stendhal à la charnière entre les deux siècles. L'Ermitage, en attirant l'attention, par le biais de sa "Chronique Stendhalienne ", sur les publications contestant l'œuvre de Stendhal, avait arrêté le front des adversaires qu'il fallait combattre, parmi lesquels figuraient notamment Albalat, Seillière, Faguet, Brunetière ; les articles stendhaliens de la Revue Blanche, pour leur part, trouvaient également leur place au sein du projet critique de la revue et dont témoignent par exemple les chroniques littéraires tenues par Muhlfeld, qui menait une bataille contre l'idée de la « littérature sociale " de Brunetière et en faveur d'un Art pour l'art opposé non seulement à l'« Art pour la Morale ou pour les Affaires", mais aussi à l'« Art tout court » ${ }^{54}$. Ainsi Suarès célèbre-t-il en Baudelaire et en Stendhal deux anti-poètes : le premier aurait mis fin « au long poème en vers, ennuyeux et inutile » et à la poésie "oratoire », en associant ses efforts à ceux de Stendhal, qui avait été le premier à oser dénoncer la rime et l'alexandrin comme «le cache-poussière de la banalité ». Le lien qui est établi entre la prose de Stendhal et la poésie de Baudelaire procède surtout de l'opposition à la poésie qui « rime pour conter, ou pour faire leçon, ou [pour] débiter des tranches d'histoire et de la géographie en tartines ${ }^{55}$; le contraste entre la forme poétique de l'un et la prose de l'autre se trouve ainsi atténué et réduit en importance aux yeux de ce poète post- 
symboliste, qui a avant tout à cœur l'autonomie de la littérature. Suarès, comme d'autres, donne du reste une lecture très partielle des réflexions de Stendhal sur le style et le langage, en privilégiant ses observations sur la rhétorique et en délaissant par exemple l'opposition que celui-ci établit entre le fond et la forme, ainsi que d'autres questions qu'il médite en disciple des Idéologues ${ }^{56}$.

L'opposition à Faguet et Brunetière n'est cependant pas le seul principe qui décide de la lecture de Stendhal par Suarès: celle-ci prend forme et s'organise également autour d'autres polémiques critiques.

Suarès attribue par exemple à l'opposition entre Stendhal et Chateaubriand une signification tout à fait particulière par rapport à celle que cette confrontation, déjà illustrée par Stendhal, acquiert sous la plume des nombreux écrivains qui la mettent en valeur au début du XX $X^{e}$ siècle.

Albert Thibaudet, en réfléchissant à cette dyade en 1930, se saisit d'une interprétation répandue lorsqu'il opère un partage dans la littérature entre deux « familles » opposées, celle émanant du Vicomte et celle descendant de Stendhal ${ }^{57}$. Thibaudet place chez les premiers Victor Hugo, Flaubert, le vicomte de Vogüé, et jusqu'à Anatole France (lequel témoigne à son avis d'un " anti-beylisme silencieux, mais profond») et, bien entendu, Maurice Barrès, cible véritable de l'article : ses ouvrages de la maturité, en dépit du titre de stendhalien qu'il s'arroge, relèveraient plus de Chateaubriand que de Stendhal. Pour étayer sa thèse, Thibaudet mentionne les jugements d'autres critiques qui ont pu révéler des traces de Chateaubriand chez Barrès, et notamment Francis Chevassu et Henri Frank ${ }^{58}$ . Il aurait bien pu évoquer Suarès aussi, qui, dans ses pages sur Stendhal, attaque en effet «la misérable postérité de Chateaubriand» et «les tigres grossiers", en visant précisément Barrès, son ennemi depuis l'Affaire Dreyfus. Suarès met en cause l'attitude de Barrès qui s'autorise abusivement de Stendhal, et il choisit de parler d'« égoïsme ", au lieu d' " égotisme ", dans la tentative peut-être de se dissocier de l'usage fait par Barrès de ce terme. La critique de la "postérité de Chateaubriand" prend cependant pour Thibaudet et pour Suarès deux significations différentes. Si Thibaudet, comme d'autres, met surtout en cause les positions politiques conservatrices de l'auteur du Culte du moi, Suarès entend avant tout plaider en faveur d'une attitude spécifiquement apolitique. Sans nier son engagement actif au moment de l'Affaire Dreyfus, Suarès conçoit, non sans paradoxe, un tel engagement comme étant voué à la défense de l'indépendance critique de l'homme de lettres, et de son détachement par rapport aux partis pris, qu'ils soient politiques ou, plus généralement, d'idées. Stendhal s'apparente pour lui à Montaigne en tant qu'esprit libre de toute prise de position nette, et comme modèle de liberté ; il en admire notamment l'attitude politique ambiguë face à la démocratie d'un côté, et à l'aristocratisme de l'autre, d'où il tire une leçon impérissable. De même, Thibaudet en appelle certes à Stendhal pour présenter une science et un art qui ne sont pas mobilisables, notamment contre Maurras qui prétend, dans sa préface à Rome, Naples et Florence, plier la leçon de Stendhal aux exigences du moment historique ${ }^{59}$. Mais si Thibaudet admet comme d'autres une analyse de l'œuvre et plus particulièrement du style de Stendhal à partir d'une perspective socio-historique, Suarès a soin au contraire d'éviter toute lecture qui pourrait avoir une connotation politique. Thibaudet s'interroge par exemple sur le style de Stendhal par rapport à la situation socio-historique de ses personnages, et notamment de Julien Sorel, qu'il appréhende comme un Tartuffe, doté en surplus d'une conscience de classe ${ }^{60}$. De même, Remy de Gourmont par exemple, dans l'article qu'il publie en 1912 sur « Le style et l'art de Stendhal », célébrait l'art de Stendhal 
par rapport aux caractères de ses personnages et au drame socio-historique qu'ils incarnent, en analysant en particulier l'hypocrisie de Julien à l'égard de sa situation sociale ${ }^{61}$. Suarès donne pour sa part une tout autre lecture du dénouement du Rouge et le noir, particulièrement atypique : à ses yeux, Julien, en tirant sur Mme de Rênal et en acceptant ainsi la prison et la mort, fait triompher l'amour sur l'argent et l'intérêt, et se dresse en héros de la passion et du désintérêt contre les visées de la société ou de la politique $^{62}$. Si la question politique rentre dans l'analyse de Suarès, c'est pour être refusée et bannie d'une œuvre d'art qui doit lui demeurer complètement étrangère.

21 La critique de Suarès envers Chateaubriand et sa descendance vise donc avant tout une idée de la littérature, celle qui compte employer l'œuvre littéraire pour dialoguer avec la cité, utiliser l'éloquence pour sauver la vie : telle est selon Suarès la faute la plus grave de la rhétorique du Vicomte (il ne sauve, de tous ses ouvrages, que les Mémoires, précisément parce qu'elles sont étrangères à ce souci), tout comme des ouvrages de Barrès et des siens, traités en "politiques à puantes racines ». La " postérité de Chateaubriand » inclut aussi dans ce sens Paul Bourget, dont la célèbre interprétation politique du Rouge et le noir à la lumière de la Commune et des aspirations de la nouvelle génération, constitue probablement la référence critique à laquelle Suarès oppose sa propre lecture du dénouement du roman.

Finalement, il faut mettre en évidence une contradiction profonde qui git au cœur de l'œuvre de Suarès, et qui se manifeste dans le rapport ambigu qu'il entretient avec Taine et avec Nietzsche lecteurs de Stendhal.

Dans un virulent article entièrement consacré à Taine, Suarès s'exprime de façon tranchante contre sa méthode : « Tout le mal vient de Taine » ${ }^{63}$, puisque ce critique aurait inauguré et transmis l'esprit de négation et de contradiction qui a un grand succès chez les " esprits faux ", les menteurs qui d'ailleurs " osent même invoquer " Stendhal au sujet de leur attitude ${ }^{64}$. Par cette critique contre Taine, Suarès entend se dissocier du stendhalisme initial et de ceux qui, parmi ses contemporains, en prolongent la vulgate. Suarès revivifie de fait au seuil de la Première Guerre mondiale un Stendhal tout à fait différent de celui célébré par les premiers stendhaliens, car il soustrait en particulier Stendhal au XVIII ${ }^{\text {e }}$ siècle auquel le rattachaient Taine (qui explique sa pensée à la lumière de la philosophie rationaliste) et Bourget (« La campagne de 1796 en Italie, et l'idéologie du XVIII ${ }^{e}$ siècle, tout Stendhal sort de ces deux influences ${ }^{65}$ ), ainsi que le faisaient déjà Sainte-Beuve ( Il procède du pur et direct XVIII ${ }^{\mathrm{e}}$ siècle ${ }^{66}$ ), et Barbey d'Aurevilly ${ }^{67}$. Suarès associe Stendhal à Montaigne plutôt qu'à Voltaire et à Rousseau, d'abord parce que ces derniers « avaient été des Français pour toute l'Europe. Stendhal le premier, depuis Montaigne, fut un Européen en France $\aleph^{68}$; ensuite, parce que Stendhal et Montaigne " sont libres même de leurs préférences. Pascal ne l'est pas; Voltaire non plus, étant si polémique de nature et trop dans l'action ${ }^{69}$. L'auteur du Voyage du condottière tient ainsi à souligner la différence qui existe entre sa propre lecture et celles, répandues même chez ses contemporains, qui minimisent l'originalité de Stendhal en la mettant sur le compte du progressisme des Lumières, ou pire qui essaient d'établir dans ce sens un lien entre Stendhal et le classicisme (dans le numéro spécial de la Revue des idées et des livres consacré à Stendhal en 1913, certains articles font de Stendhal un écrivain classique afin de le présenter comme une gloire nationale ${ }^{70}$ ). Stendhal est actuel, selon Suarès, du fait de ses sempiternelles leçons qui échappent à toute appartenance à une époque donnée : «il pense de façon à vivre encore parmi nous ", précise-t-il dans un article où il l'oppose à cet enfant du XVIII ${ }^{e}$ siècle qu'est selon lui Casanova ${ }^{71}$. 

contestation, il en partage incontestablement les présupposés, comme en témoigne son œuvre totalement élaborée dans le souci de l'audace et du non-conformisme. Et en ce qui concerne tout particulièrement Stendhal, Suarès ne peut qu'être d'accord avec les observations de Taine, lequel, à l'encontre du jugement de Sainte-Beuve sur Stendhal, est le premier à faire l'apologie du "naturel charmant» de son style, opposé à celui des auteurs tragiques, « orateurs » et « rhétoriciens $~^{72}$. Suarès condamne en réalité, non sans ambiguité, une attitude qu'au fond il approuve : on retrouve la même contradiction dans sa critique envers Nietzsche.

Taxé souvent de nietzschéisme par la critique de son époque ${ }^{73}$, Suarès écrit des morceaux très polémiques sur le philosophe allemand, et surtout il prend position contre l'assimilation entre celui-ci et Stendhal, qui constitue au seuil $\mathrm{du} \mathrm{XX}^{\mathrm{e}}$ siècle un poncif critique autant pour les détracteurs de Stendhal (Faguet, Seillière ${ }^{74}$ ) que pour les stendhaliens (Jean Mélia et Léon Blum entre autres ${ }^{75}$ ). À l'encontre de ceux qui s'approchent de Stendhal par le biais du philosophe allemand en le proclamant son précurseur $^{76}$, Suarès s'attache à regarder Nietzsche à la lumière de Stendhal, pour le découvrir "plutôt prêtre ", moraliste en quelque sorte, et nettement moins libre que très occupé de tout renier ${ }^{77}$. C'est que l'actualité que Suarès assigne à Stendhal procède plus de la défense et de l'illustration d'une conception de la littérature, que de la lutte du début du siècle contre le kantisme du monde universitaire et contre la philosophie morale, combat qui contribue par ailleurs de façon substantielle au renouveau d'intérêt pour Stendhal. Il reste que Suarès fait grief à Nietzsche d'une volonté négatrice, d'un ton résolu et passionné, d'une fonction critique exercée d'une manière intégrale, qui ne sont pas tout à fait étrangers à son œuvre elle-même. On a essayé d'expliquer la violence des écrits de Suarès contre Nietzsche par l'auto-identification dont le poète fait preuve à l'égard de ce dernier ${ }^{78}$; comme pour Taine, Suarès met en question devant le philosophe allemand les présupposés de sa propre œuvre, qu'il essaie de soustraire à toute certitude critique et à la tentation négatrice tout court. Aux côtés de Stendhal, et sous le signe de cette divinité protectrice, Suarès tente de rompre et de quitter les rangs établis, ainsi que de transcender tout parti pris, même le sien.

Accompagné par Stendhal, c'est-à-dire à la fois sous son signe et suivant son modèle, Suarès dialogue donc avec son temps et conduit ses batailles littéraires. L'analyse de l'œuvre de Stendhal prend forme à partir d'une idée précise de la littérature, qu'il est possible de cerner en examinant les polémiques que Suarès entretient de façon plus ou moins explicite avec la critique qui lui est contemporaine. En enfant du symbolisme et en poète, Suarès peut défendre une prose et un style qui affichent le dédain de la forme, poussé par le besoin de défendre l'ensemble des priorités de sa conception de la littérature, contre les attaques qui leur sont livrées: ainsi les paradoxes d'un Stendhal apparenté à Baudelaire et Verlaine sont-ils possibles, tout comme ceux d'une critique tainienne contre Taine et nietzschéenne contre Nietzsche. La figure du paradoxe constitue la véritable clé de la lecture de Stendhal proposée par Suarès : non seulement parce que ce dernier présente en termes paradoxaux le maître et son œuvre, en s'appuyant sur la position effectivement singulière occupée par Stendhal dans l'histoire littéraire, à savoir celle d'un auteur romantique contre les classiques et classique contre les romantiques; mais aussi parce que Suarès énonce les apories qui gisent au cœur de sa conception de la littérature en les reportant sur Stendhal, et en les légitimant de la sorte. Sa démarche rejoint ainsi parfaitement celle de Gide, qui avance de façon analogue, dans

Revue italienne d'études françaises, 2 | 2012 
son célèbre «Billet à Angèle » de mars 1921, une lecture du classicisme et en particulier de Stendhal, explicitement évoqué, en tant que caractérisé par des paradoxes, celui du «romantisme dompté » et celui de l'«individualisme dans le renoncement à l'individualité » entre autres ${ }^{79}$. C'est ainsi que Suarès participe complètement de l'« esprit $N R F$ ", auquel il est si rarement associé, et qu'il contribue pourtant si activement à constituer.

\section{NOTES}

1. Cf. les lettres de Gide du 24 novembre 1909 et du 12 novembre 1910 au sujet de la collaboration de Suarès à la NRF, dans A. Gide - A. Suarès, Correspondance 1908-1920, préface et notes de S. D. Braun, Paris, Gallimard, 1963; et la lettre du 12 février 1912, où Gide se félicite du succès de Copeau qui a réussi à engager Suarès pour une collaboration régulière.

2. Suarès publiera 36 articles entre avril 1926 et juin 1940. Cf. aussi J. Paulhan - A. Suarès, Correspondance 1940-1948, textes établis et préfacés par Y.-A. Favre, Mortemart, Rougerie, 1992.

3. A. Suarès, «D'après Stendhal », dans Nouvelle Revue française (dorénavant NRF), n. 65 et n. 66, mai et juin 1914, p. 853-871 et p. 998-1034. Cet article est repris dans l'un des deux recueils où Michel Drouin a réuni quelques-uns des essais littéraires de Suarès: Portraits et préférences, édition établie, présentée et annotée par M. Drouin, Paris, Gallimard, 1991, p. 55-90 (l'autre recueil est : A. Suarès, Âmes et visages, XIII ${ }^{e}$ XVIII ${ }^{e}$ siècle, édition établie, présentée et annotée par M. Drouin, Paris, Gallimard, 1989). La version reproduite dans ce recueil étant cependant incomplète du fait qu'elle comporte un choix des pages, nous ferons référence à l'article originaire paru dans la NRF pour les citations et les notes qui suivent.

4. Cf. le jugement porté par A. Gide, Journal, 1889-1939, Paris, Gallimard, 1941, 27 juin 1908, p. 269, sur la « Visite à Pascal » que Suarès publie en 1900 dans la Revue des deux mondes, et aujourd'hui reproduite dans Id., Âmes et visages, cit., p. 122-160.

5. Sur les rapports Suarès-Stendhal ont été jusqu'à présent publiés l'article de R. Parienté, "André Suarès dans le sillage de Stendhal », dans Stendhal Club, n. 136, 15 juillet 1992, p. 307-317, et celui de G. Jessula et M. Drouin, « Encore André Suarès », ibid., n. 141, 15 octobre 1993, p. 24-34. 6. A. Suarès, Voyage du condottière, Paris, Granit, 1984.

7. A. Suarès, «Toujours Stendhal », dans Id., Xénies, Paris, Émile-Paul frères, 1923 (édition préoriginale partielle dans Les Écrits nouveaux, 1919-1920). Aujourd'hui dans Id., Idées et visions et autres écrits polémiques, philosophiques et critiques, 1897-1923, édition établie par R. Parienté, Paris, Laffont, 2002, p. 948-951.

8. A. Suarès, «Stendhal en Lombardie », dans Id., Voyage du condottière, cit., p. 91.

9. Cette partie du Voyage, "Vers Venise ", a été écrite après les trois voyages de Suarès en Italie en 1895, 1902 et 1909, et a été publiée chez Cornély en 1910.

10. A. Gide, Journal (1899-1939), Paris, Gallimard, 1941, p. 255, 8 décembre 1907.

11. Ibid., p. 387, 19 mai 1913.

12. A. Gide, «Journal sans dates », dans NRF, $1^{\text {er }}$ janvier 1910, aujourd'hui dans Id., Essais critiques , édition présentée, établie et annotée par P. Masson, Paris, Gallimard, 1999, p. 206-215 : «je sais bien que, par contre, cette égalité de l'esprit lui refuse les sublimes sursauts du lyrisme; mais mon esprit ne me suggère cela que pour excuse de n'être pas plus égal ».

13. Ibid., p. 816. 
14. J. Rivière, lettre à Gide, 2 août 1910, dans A. Gide - J. Rivière, Correspondance : 1909-1925, édition établie, présentée et annotée par P. de Gaulmyn et A. Rivière, Paris, Gallimard, 1998, p. 133-134.

15. R. de Gourmont, «Le style et l'art de Stendhal », dans Le Temps, 19 mai 1912, aujourd'hui dans Stendhal, préface de M. Crouzet, Paris, Presses de l'Université Paris-Sorbonne, « Mémoire de la critique ", 1996, p. 497-502.

16. Alain, Propos, texte établi et présenté par Maurice Savin, préf. d'André Maurois, Paris, Gallimard, 1956, 14 novembre 1923, p. 548-549, et Id., Stendhal et autres textes, textes réunis et présentés par F. Foulatier, Paris, Presses Universitaires de France, 1994.

17. J. Prévost, La création chez Stendhal, Paris, Mercure de France, 1951.

18. Cf. Ph. Berthier et É. Bordas (dir.), Stendhal et le style, Paris, Presses de la Sorbonne Nouvelle, 2005, notamment les articles de G. Kliebenstein, «Stendhal et la rhétorique », p. 31-52, et de Y. Ansel, « Politique du style », p. 75-89.

19. Cf. H. Rochefort, Les Aventures de ma vie, Paris, Dupont, 1896-1898, 5 vol. , t. II, p. 54.

20. G. Flaubert, lettre à Louise Colet, 22 novembre 1852, dans Id., Correspondance, t. II, Paris, Gallimard, « Bibliothèque de la Pléiade », 1980, p. 179.

21. P. Valéry, «Stendhal » (1927), dans Id., CEuvres, édition établie et annotée par J. Hytier, introduction biographique par A. Rouart-Valéry, 2 vol. , Paris, Gallimard, 1957 et 1960, t. I, p. 582. 22. A. Albalat, Le Travail du style enseigné par les corrections manuscrites des grands écrivains, Paris, Colin, 1903.

23. Cf. H. Cordier, Stendhal et ses amis, Paris, impr. de C. Hérissey, 1890, et A. Chuquet, StendhalBeyle, Paris, Plon, 1902.

24. A. Suarès, «Stendhal en Lombardie », cit., p. 90-91.

25. Id., « Toujours Stendhal », cit., p. 950-951.

26. Stendhal, lettre à Balzac du 16 octobre 1840, Correspondance générale, V. Del Litto (éd.), Paris, Champion, 1999, p. 404.

27. A. Suarès, « Toujours Stendhal », cit., p. 950-951, et Id., «Stendhal », Valeurs II, in Id., Valeurs et autres écrits historiques, politiques et critiques, 1923-1948, édition établie par R. Parienté, Paris, Laffont, 2002, p. 754.

28. Stendhal, « Marginalia ", dans La Revue Blanche, 1901, t. XXVI, p. 274-286.

29. La Revue Blanche publie à partir de 1893 des inédits de Stendhal à raison à peu près d'un par an: les pages de deux romans non aboutis, donnés par Stryenski, mars 1893 ; "Avis aux têtes légères » et «Le procès de Julien Sorel », mars 1894 ; "Consultation pour Banti ", $1^{\text {er }}$ octobre 1897 ; « Burrhus », 15 octobre 1897 ; lettre au comte Cini, t. XVIII, 1899.

30. Léon Blum collabore à la Revue Blanche à partir de juillet 1892 ; il consacre à Stendhal quelques pages de ses "Nouvelles conversations avec Eckermann", rubrique anonyme tenue à partir de mai 1894, et un compte rendu dans le numéro du 15 novembre 1897, sur le Napoléon de Stendhal publié par J. de Mitty.

31. P. Léautaud, «Le Stendhal-Club», dans L'Ermitage, $16^{\mathrm{e}}$ année, n. 3, 1905, aujourd'hui dans Le Temps du Stendhal-Club : 1880-1920, textes réunis par Ph. Berthier et G. Rannaud, Toulouse, Presses universitaires du Mirail, 1994, p. 27.

32. Cf. P. Léautaud, Journal littéraire, 4 vol. , Paris, Mercure de France, 1986, t. I (novembre 1893juin 1928), p. 47 : «Il faut des livres de faits clairs et nets, écrits "net et court", comme écrivait dernièrement Régnier sur Stendhal » (1902).

33. P. Léautaud, « Le Stendhal-Club», cit., p. 34.

34. A. Suarès, « Stendhal ", Valeurs II, cit., p. 754.

35. Cf. Coffe, «Chronique Stendhalienne », L'Ermitage, 1905, vol. XXXII, p. 243 sq (2 ème article), et A. Suarès, «Stendhal en Lombardie », cit, p. 94-95.

36. La «Chronique Stendhalienne» se compose de quatre longues publications, parues dans L'Ermitage entre mai et juillet 1905 (vol. XXXII et XXXIII), et ensuite recueillies dans le volume Chronique Stendhalienne, Milano, Coffe et Cie, 1907. 
37. Voir A. Suarès, "Molière II ", dans Âmes et visages, cit., p. 119, et Id., «Bossuet ", ibid., p. 161-169.

38. A. Suarès, « Chateaubriand », dans Portraits, cit., p. 36.

39. Id., « D’après Stendhal », cit., p. 1002-1004 ; cf. aussi Id., « Musset », dans Portraits et préférences , cit., p. 117.

40. A. Suarès, « D’après Stendhal », cit., p. 998 sq.

41. Ibid., p. 1028.

42. A. Suarès, "Stendhal, Verlaine, Gérard de Nerval et autres gueux " (1925), aujourd'hui dans Id., Valeurs, cit., p. 80-82.

43. A. Suarès, « D’après Stendhal », cit., p. 859.

44. A. Suarès, « Verlaine », dans Id., Portraits et préférences, cit., p. 222.

45. A. Suarès, « D’après Stendhal », cit., p. 1025.

46. A. Gide, "Journal sans dates" (décembre 1910), dans Id., Essais critiques, cit., p. 261-263 : «D'où vient que le mensonge de Stendhal m'amuse; tandis que celui de Chateaubriand m'exaspère jusqu'à me rendre la lecture de ses Voyages ou de ses Mémoires parfois impossible?».

47. Id., "Préface à Armance ", ibid., p. 544.

48. Id., « Rimbaud », ibid., p. 314.

49. Id., «Baudelaire et M. Faguet », paru dans la NRF du $1^{\mathrm{er}}$ novembre 1910, et aujourd'hui dans Id., Essais critiques, cit., p. 255 et p. 250.

50. A. Gide - A. Suarès, Correspondance, cit., lettre du 3 novembre 1910, p. 43-44.

51. A. Suarès, « D'après Stendhal », cit., p. 1013.

52. F. Brunetière, appréciation sur la dissertation de Rolland sur Stendhal en 1887, dans G. Salamand, «Romain Rolland et Stendhal, textes inédits », Stendhal Club, n. 102, 15 janvier 1984, p. 121-129.

53. Sur le rapport complexe qui lie Suarès à son ancien professeur, cf. A. Suarès, « $M$. Brunetière ", dans Id., Idées et visions, cit., p. 200-205.

54. Cf. en particulier L. Muhlfeld, «Chronique de la littérature », dans Revue Blanche, janvier 1892.

55. A. Suarès, « Baudelaire et Les Fleurs du mal », dans Portraits et préférences, cit., p. 165.

56. Cf. Y. Ansel, « Politique du style », cit., et M. Crouzet, Stendhal et le langage, Paris, Gallimard, 1981.

57. A. Thibaudet, «Les deux rives ", dans Id., Réflexions sur la littérature, édition établie et annotée par A. Compagnon et Ch. Pradeau, Paris, Gallimard, 2007, p. 1342-1350.

58. Ibid., p. 1347-1348.

59. Cf. C. Maurras, «Stendhal contemporain ", préface à Stendhal, Rome, Naples et Florence, Paris, Champion, 1919, p. I-XXXIV ; A. Thibaudet, « Sur la démobilisation de l'intelligence » (1920), dans Id., Réflexions sur la politique, édition établie par A. Compagnon, Paris, Laffont, 2007, p. 254-262.

60. A. Thibaudet, "Stendhal et Molière ", dans NRF, $1^{\text {er }}$ novembre 1923, aujourd'hui dans Id., Réflexions sur la littérature, cit., p. 963-964.

61. R. de Gourmont, « Le style et l'art de Stendhal », cit..

62. A. Suarès, "D'après Stendhal", cit., p. 865-866: "Julien et l'adorable Rênal, quoi qu'ils fassent, étaient voués à la même vie et à la même mort d'amour : l'amour est leur fatalité. Pour être tout à son amour, il [Julien] se sépare de la vie. Il tue son ambition, pour se rendre sans partage à sa maîtresse. [...] En Julien Sorel, l'ambition n'était que le masque de la passion, et le moindre usage d'une nature conquérante ».

63. A. Suarès, « Contre Taine », dans La Grande Revue, 10 mars 1909, aujourd'hui dans Id., Portraits et préférences, cit., p. 171-180.

64. Ibid., p. 174.

65. P. Bourget, « La genèse du roman contemporain » (1878), dans Stendhal, Mémoire de la critique, cit., p. 425. 
66. C.-A. Sainte-Beuve, «M. de Stendhal. Ses Cuvres complètes », (1854), aujourd'hui dans Stendhal, Mémoire de la critique, cit., p. 297-326.

67. J. Barbey d'Aurevilly, « De Stendhal » (1856), ibid., p. 375-384.

68. A. Suarès, « D’après Stendhal », cit., p. 1033.

69. A. Suarès, « Toujours Stendhal », cit., p. 950.

70. Le numéro de mars 1913 de la Revue critique des idées et des livres comprend notamment des articles d'Albert Guinon, Henry Bordeaux, Pierre Lasserre, Fagus, aux côtés d'articles plus scientifiques donnés par des stendhaliens tels que Henri Cordier et Adolphe Paupe.

71. A. Suarès, « Casanova », dans Âmes et visages, cit., p. 258.

72. H. Taine, «Stendhal (Henri Beyle)», dans Nouvelle Revue de Paris, $1^{\mathrm{er}}$ mars 1864, aujourd'hui dans Stendhal, Mémoire de la critique, cit., p. 393-413.

73. Cf. C. Mauclair, "André Suarès et son dernier livre Voici l'homme », dans La Grande revue, 25 avril 1907 ; P. Souday, Les livres du temps, $1^{\text {ère }}$ série, Paris, Émile-Paul, 1929, p. 288-297.

74. Cf. É. Faguet, Politiques et moralistes du XIXe siècle, Paris, Lecène-Oudin, 1891-1900, III série : «Stendhal comme le premier des nietzschéens ».

75. Cf. L. Blum, Stendhal et le beylisme [1914], Paris, Albin Michel, 1947, p. 123 : «En dépit de toutes les différences, le beylisme repose sur une vue analogue à celle de Nietzsche». Cf. James S. Schields, «Le syndrome de Nietzsche dans la critique stendhalienne ", dans Le Temps du StendhalClub, cit., p. 75-89.

76. Cf. F. Nietzsche, Par-delà bien et mal. Prélude d'une philosophie de l'avenir, textes et variantes établis par G. Colli et M. Montinari, traduits de l'allemand par C. Heim, Paris, Gallimard, 1971, p. 175: «[...] Henri Beyle, cet extraordinaire précurseur qui parcourût à une allure napoléonienne, en veneur et en découvreur, l'Europe de son temps, et plusieurs siècles de l'âme européenne : il a fallu deux générations pour le rattraper tant bien que mal, pour deviner après lui quelques-unes des énigmes qui le tourmentèrent et qui le ravirent, cet étonnant épicurien, ce point d'interrogation fait homme, le dernier grand psychologue de la France ».

77. A. Suarès, « Excuse à Nietzsche ", dans Id., Valeurs, cit., p. 94-120.

78. J. Lecarme, "Suarès et Nietzsche ", dans Suarès et l'Allemagne, textes réunis et présentés par Y.-A. Favre, Paris, Minard, 1977, p. 47-68.

79. A. Gide, « Billet à Angèle (mars 1921) », dans Essais critiques, cit., p. 281-283.

\section{INDEX}

Mots-clés : Stendhal, Suarès (André), style et rhétorique, Nouvelle Revue Française, histoire littéraire 